\title{
Nonlinear transport of Bose-Einstein condensates through waveguides with disorder
}

\author{
Tobias Paul, ${ }^{1}$ Patricio Leboeuf, ${ }^{2}$ Nicolas Pavloff, ${ }^{2}$ Klaus Richter, ${ }^{1}$ and Peter Schlagheck ${ }^{1}$ \\ ${ }^{1}$ Institut für Theoretische Physik, Universität Regensburg, 93040 Regensburg, Germany \\ ${ }^{2}$ Laboratoire de Physique Théorique et Modèles Statistiques, Université Paris Sud, Bâtiment 100, F-91405 Orsay Cedex, France
}

(Received 19 September 2005; published 28 December 2005)

\begin{abstract}
We study the coherent flow of a guided Bose-Einstein condensate incident over a disordered region of length $L$. We introduce a model of disordered potential that originates from magnetic fluctuations inherent to microfabricated guides. This model allows for analytical and numerical studies of realistic transport experiments. The repulsive interaction among the condensate atoms in the beam induces different transport regimes. Below some critical interaction (or for sufficiently small $L$ ) a stationary flow is observed. In this regime, the transmission decreases exponentially with increasing $L$. For strong interaction (or large $L$ ), the system displays a transition toward a time-dependent flow with an algebraic decay of the time-averaged transmission.
\end{abstract}

DOI: 10.1103/PhysRevA.72.063621

PACS number(s): 03.75.Kk, 72.15.Rn, 42.25.Dd

\section{INTRODUCTION}

The extraordinary experimental control achieved over atomic Bose-Einstein condensates (BECs) provides new testgrounds for phenomena coming from many different fields. On the one hand these systems allow extensive study of nonlinear phenomena such as four-wave mixing [1], propagation of bright [2] and dark [3] solitons, or the dynamics of Bloch oscillations in the presence of atom-atom interactions $[4,5]$. On the other hand the rapid progress in this field has led to a number of fascinating experiments probing complex condensed matter phenomena, such as the Mott transition in optical lattices [6], the creation of vortices [7], the Josephson effect [8] or the BEC-BCS crossover [9]. Bose-Einstein condensates link these two prominent fields of current research in an exciting and unique way.

Wave mechanical transport in atomic vapors appears as a new direction for these trans-disciplinary studies that provide deeper insights into transport phenomena in the presence of interaction. Indeed, BEC systems are intrinsically phase coherent, as are the clean two-dimensional electronic structures studied in mesoscopic physics at low temperatures. In addition, interaction is much more simply modeled in BEC systems than the electrostatic electron-electron potential and its sign (repulsive or attractive) and strength can be tuned almost at will. The link between matter-wave physics and electronic transport phenomena became ultimately apparent with the advent of microscopic traps and waveguides for atoms, known as atom chips [10-12]. Related studies include the attempt to generalize Landauer's theory of conductance to cold atoms [13], the atom blockade phenomenon in quantum-dot-like potentials [14], as well as nonlinear resonant transport of Bose-Einstein condensates [15], to mention just a few examples.

A new direction in this context is the transport of BoseEinstein condensates through disordered potentials. A relevant question is to what extent a Bose-Einstein condensate is subject to Anderson localization [16,17] in the presence of disorder, as well as how this scenario is affected by the atomatom interaction. There is a growing interest in the BEC community in issues related to the behavior of matter waves in disordered potentials. It started with the observation of "fragmentation of the condensate" over a microchip [18]. Nowadays a random potential is routinely engineered using an optical speckle pattern and its effects on the expansion of the condensate have been explored in Refs. [19,20].

In contrast to studies where the condensate is initially at rest, we focus in the present paper on the effect of disorder on a propagating Bose-Einstein condensate. In an adiabatic approximation, the dynamics reduces to an effective onedimensional (1D) transport problem; this is the so-called 1D mean-field regime [21]. We furthermore assume that the mean kinetic energy of the atoms in the condensate is larger than the typical height of the barriers induced by the disorder potential, i.e., perfect transmission is expected by classical mechanics. For the sake of concreteness, we restrict ourselves to one specific type of disorder: the one experienced by a condensate that is magnetically trapped above a corrugated microchip. To this end we introduce a model that could be characterized as a "dirty-wire model" where the current in the microfabricated wire has white-noise fluctuations. This simple model captures most of the characteristics of the random potentials observed over corrugated microchips. We point out, however, that our results are not expected to be sensitive to the particular type of disorder, as long as the latter is sufficiently smooth and can be characterized by a well-defined correlation length.

Previous theoretical studies of the effect of disorder on the transmission of nonlinear waves mainly focused on attractive interaction and looked for stationary solutions of the problem (see the review [22]). Then, one has to choose between fixed input and fixed output boundary conditions. The latter case is less realistic, but simpler to discuss: It leads to algebraic decay of the transmission [23]. The former case is complicated by the advent of multistability. However, the results of Knapp et al. [24] show that, for short sample size, the mean transmission is poorly affected by a weak nonlinearity (as compared to the linear case), whereas for larger samples and stronger nonlinearity, evidence of delocalization is found.

Realistic transport processes of Bose-Einstein condensates are different from the above-mentioned studies because they typically involve particles experiencing repulsive interactions. We will see below that in this case the assumption of stationarity is not appropriate because for large disordered 
regions or strong nonlinearity stationary solutions are dynamically unstable. In typical experiments the population of a given final state can only be achieved through a timedependent process (such as the gradual filling of an initially empty waveguide with matter waves). As a result, if a stationary scattering state is unstable, the transport properties of the condensate may be unrelated to the transmission coefficient associated with that state, whereas a study of stationary flows might misleadingly give some weight to this state (if the transmission is averaged over all possible stationary solutions for instance).

We thus consider a setup that is relevant to experimental realizations and adapted to this specific transport scenario explicitly taking into account the possibility of timedependent scattering: a coherent source of atoms emits matter waves that propagate in the magnetic waveguide and encounter on their path a disorder region of length $L$. We show that the presence of a repulsive atom-atom interaction has dramatic effects on the transport properties of the condensate. As is the case for attractive nonlinearity, Anderson localization is observed only in the regime of small interaction strengths and sample lengths. In this regime the transmission decreases exponentially with increasing $L\left[\propto \exp \left(-L / L_{l o c}\right)\right]$, with a localization length $L_{l o c}$ modified by the interaction. For large sample lengths or strong interaction, time-dependent scattering processes occur. In contrast to the previous regime, one observes an Ohmic decrease of the time-averaged transmission $\left(\propto L^{-1}\right)$.

The paper is organized as follows. In Sec. II we set up the theoretical framework that is necessary to study transport through mesoscopic waveguides, introduce an effective onedimensional Gross-Pitaevskii equation, and present a numerical method that is particularly suited to study transport processes of Bose-Einstein condensates in waveguides. In Sec. III we introduce a one-dimensional model for the random magnetic potential along the center of the waveguide. We will show that a microscopic meandering of the current in the wire on the atom chip leads to a Lorentzian-correlated random potential. In Sec. IV we investigate the regime of weak disorder potentials and give a simple analytic expression for the condensate wave function in the guide. In Sec. V we discuss numerical results for transport through moderateand strong-disorder regions. We consider in particular the scaling of the transmission with the length of the disorder region. The paper closes with some concluding remarks. Some technical points are given in the appendixes. In Appendix A we derive a relation between the mean transmission and the correlation function of the disorder potential. In Appendix $\mathrm{B}$ we rederive, using standard WKB techniques, a result that is obtained heuristically in the main text.

\section{TRANSMISSION THROUGH WAVEGUIDES}

We consider a coherent beam of Bose-Einstein-condensed atoms at zero temperature, propagating through a cylindrical magnetic waveguide of axis $x$. The condensate is formed by atoms of mass $m$ which interact via a two-body potential characterized by its 3D $s$-wave scattering length $a_{s c}$. We consider the case of repulsive effective interaction, i.e., $a_{s c}>0$.
The condensate is confined in the transverse direction by a harmonic potential of pulsation $\omega_{\perp}$. This transverse confinement is characterized by the harmonic oscillator length $a_{\perp}$ $=\left(\hbar / m \omega_{\perp}\right)^{1 / 2}$.

In the following we restrict ourself to the $1 \mathrm{D}$ mean-field regime [21] corresponding to a density range such that $\left(a_{s c} / a_{\perp}\right)^{2} \ll n_{1 \mathrm{D}} a_{s c} \ll 1$, where $n_{1 \mathrm{D}}$ denotes the typical order of magnitude of the $1 \mathrm{D}$ density $n(x, t)$ of the system. The first of these inequalities ensures that the system does not get into the Tonks-Girardeau limit and the second that the transverse wave function is the ground state of the linear transverse Hamiltonian; see, e.g., the discussion in Refs. [21,25]. In this regime the system is described by a $1 \mathrm{D}$ order parameter $\psi(x, t)$ [such that $n(x, t)=|\psi(x, t)|^{2}$ ] depending only on the spatial variable $x$ along the guide. $\psi(x, t)$ obeys the 1D Gross-Pitaevskii equation

$$
i \hbar \frac{\partial \psi}{\partial t}=\left(-\frac{\hbar^{2}}{2 m} \frac{\partial^{2}}{\partial x^{2}}+V(x)+g n(x, t)\right) \psi,
$$

with $g=2 \hbar \omega_{\perp} a_{s c}$ [26-28]. $V(x)$ is an effective onedimensional potential along the waveguide, to which the condensate is exposed during the propagation process. We will see in Sec. III how it may originate from irregularities of a wire used for creating the magnetic confinement.

In the absence of a potential $[V(x) \equiv 0]$ the plane wave

$$
\psi(x, t)=\sqrt{n_{0}} \exp (i k x-i \mu t / \hbar)
$$

is obviously a solution of the Gross-Pitaevskii equation (1). It satisfies the dispersion relation

$$
\mu=\frac{m}{2} \frac{J^{2}}{n_{0}^{2}}+g n_{0},
$$

where the particle current is given by $J=n_{0} \hbar k / m$. Therefore, the chemical potential $\mu$ and the equilibrium constant density $n_{0}$ of a freely propagating condensate beam are determined by the current $J$, the wave vector $k$, and the effective interaction strength $g$. At this point, we mention that it was demonstrated in [28] that Eq. (3) exhibits two constant-density solutions: a low-density (supersonic) one and a high density (subsonic) one, where the transport is respectively dominated by the kinetic energy or by mutual interaction of the atoms. Both solutions are plane waves of the form $\psi(x)=A e^{i k x}$, but with different wave vectors $k$ and particle densities $A^{2}$. As we are considering rather small condensate densities and large velocities in the waveguide, the supersonic solution will be the relevant one in the context of this paper.

We now assume the presence of a disorder potential $V(x)$ in the waveguide which is finite between $x=0$ and $L$ and vanishes elsewhere. In this case, a BEC that is injected into the initially condensate-free disorder region from the upstream side (i.e., at $x<0$ ) in general does not freely propagate to the downstream region (at $x>L$ ), but undergoes a scattering process. In this paper we shall compute transport properties of a system where a monochromatic beam of condensate with well-defined current $J_{i}$ is injected into the disorder region (see Fig. 1). This means, we consider the propagation process in terms of a so-called fixed input problem $[22,24]$. 


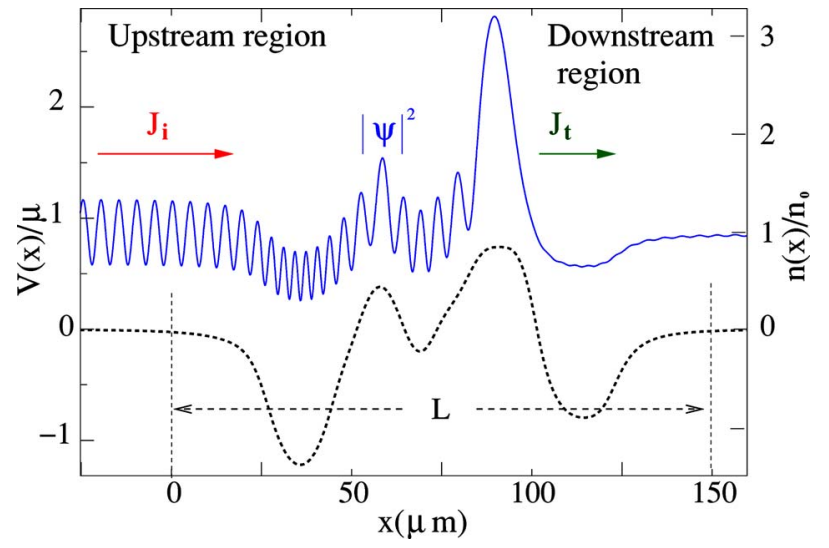

FIG. 1. (Color online) A condensed beam with incident current $J_{i}$ can populate a stationary scattering state. The solid line shows its longitudinal density $n(x)$ (in units of the equilibrium density $n_{0}$ ). In the downstream region, $\psi$ tends to a plane wave with transmitted current $J_{t}$. The dashed line displays the scattering potential $V(x)$ in units of the chemical potential $\mu$.

Our purpose is now to compute transmission coefficients for the condensate transport through the disordered region. Furthermore, we shall investigate to what extent it is possible to populate stationary scattering states, i.e., stationary solutions $\psi(x, t)=\psi(x) \exp (-i \mu t / \hbar)$ satisfying the outgoing boundary condition $\psi(x)=\sqrt{n_{0}} e^{i k x}$ (with $k>0$ ) for $x \rightarrow+\infty$, where $n_{0}$ is the density associated with the supersonic solution [29]. This question can be addressed by integrating the time-dependent Gross-Pitaevskii equation (1) in the presence of a source term that is localized in the upstream region and emits monochromatic matter waves. Such a source models the coupling of the waveguide to a reservoir of condensate from which matter waves are injected into the guide. It has been demonstrated in [15] that this approach is particularly well suited to compute transmissions for fixed input problems. Additionally, it allows one to determine for a given potential $V(x)$ whether an incident monochromatic beam populates a stationary scattering state or not.

Hence, we consider now the modified Gross-Pitaevskii equation with a source that is localized at the position $x_{0}$ in the upstream region,

$$
\begin{aligned}
i \hbar \frac{\partial \psi(x, t)}{\partial t}= & \left(-\frac{\hbar^{2}}{2 m} \frac{\partial^{2}}{\partial x^{2}}+V(x)+g|\psi(x, t)|^{2}\right) \psi(x, t) \\
& +S_{0} \exp (-i \mu t / \hbar) \delta\left(x-x_{0}\right)
\end{aligned}
$$

$S_{0}$ is the source amplitude which determines the emitted current. To understand the functionality of the source term, it is instructive to consider first solutions of Eq. (4) in the absence of the potential $V(x)$. In this case there exist plane-wave solutions with constant density $n$. To demonstrate this, we switch to the Fourier space, where Eq. (4) takes the form (for constant $n$ )

$$
\left(\frac{i}{\hbar} \frac{\partial}{\partial t}-\frac{\hbar^{2} q^{2}}{2 m}-g n\right) \tilde{\psi}(q, t)=S_{0} e^{-i q x_{0}} e^{-i \mu t / \hbar} .
$$

This equation admits a solution of the form

$$
\tilde{\psi}(q, t)=\frac{S_{0} e^{-i q x_{0}} e^{-i \mu t / \hbar}}{\mu-g n-\hbar^{2} q^{2} /(2 m)} .
$$

By transforming back to real space, we find that the source emits in both directions the monochromatic wave

$$
\psi(x, t)=\frac{S_{0} m}{i k \hbar^{2}} e^{i k\left|x-x_{0}\right|} e^{-i \mu t / \hbar}
$$

In Eq. (7) $k$ is self-consistently defined by $(\hbar k)^{2}=2 m[\mu$ $\left.-g\left|S_{0}\right|^{2} m^{2} /\left(\hbar^{4} k^{2}\right)\right]$. The current emitted by the source can be calculated by evaluating the quantum mechanical current operator. We find $J_{i}= \pm\left|S_{0}\right|^{2} m /\left(\hbar^{3} k\right)$ ( + for $x>x_{0}$; - for $x<x_{0}$ ).

We now return to the general case $V(x) \neq 0$. In order to perform the numerical integration, the wave function $\psi(x, t)$ is expanded on a finite lattice and is propagated in the real time domain. As we are dealing with an open system, artificial backscattering at the boundaries of the lattice has to be avoided. For that purpose we impose absorbing boundary conditions that are well suited for transport problems [30] and can be generalized to account for weak or moderate nonlinearities [31].

As in real experiments we choose as initial condition $\psi(x, t=0) \equiv 0$. In order to compute the condensate wave function we numerically integrate $\psi(x, t)$ in Eq. (4) while adiabatically tuning the source amplitude $S_{0}$ from 0 up to a given maximum value that corresponds to a desired incident current $J_{i}$. This approach simulates a realistic propagation process, where a coherent Bose-Einstein condensate beam with chemical potential $\mu$ is injected into the initially empty waveguide from a reservoir. For comparatively weak nonlinearities a stationary scattering state of the form $\psi(x, t)$ $=\psi(x) e^{-i \mu t / \hbar}$, which corresponds to a supersonic solution in the downstream region, is generally obtained from the numerical propagation. This stationary wave function satisfies the time-independent Gross-Pitaevskii equation

$$
\mu \psi(x)=\left(-\frac{\hbar^{2}}{2 m} \frac{\partial^{2}}{\partial x^{2}}+V(x)+g|\psi(x)|^{2}\right) \psi(x) .
$$

In contrast to the case of the linear Schrödinger equation the transmission coefficient cannot be computed by simply decomposing the upstream wave function into an incident and a reflected part because the superposition principle is not valid in the presence of the nonlinear term. Such a decomposition is only possible in the limit of small interaction strengths or small back reflections [32]. However, our numerical approach permits nevertheless a straightforward access to the transmission coefficient also in the nonlinear case. The latter is evaluated by the ratio of the current $J_{t}$ in the presence of the potential $V(x)$ (i.e., the transmitted current) to the current $J_{i}$ obtained in the absence of $V(x)$ (the incident current that is emitted by the source). This approach provides a natural extension of the usual definition of transmission coefficients in quantum mechanics to the nonlinear case [15].

In the nonlinear regime, due to dynamical instabilities the wave function $\psi(x, t)$ does not always converge toward a stationary state but can remain time dependent (cf. Sec. V). In that case, the downstream current is no longer constant 


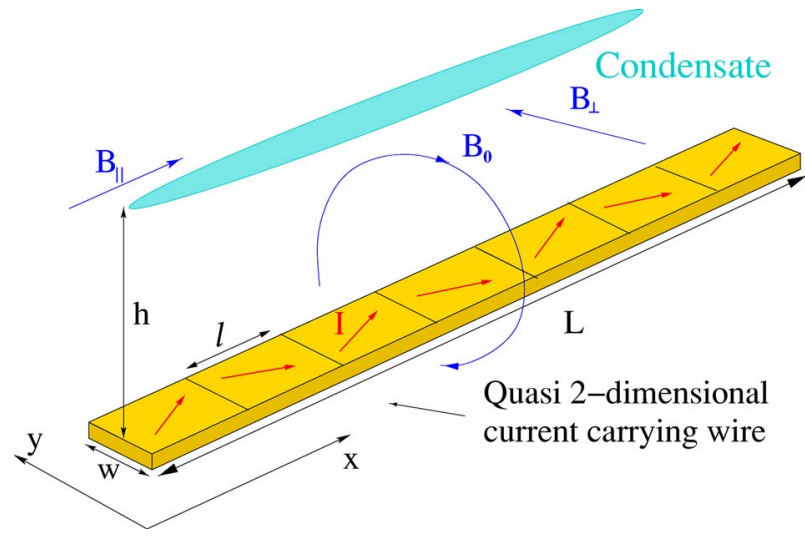

FIG. 2. (Color online) Main building block to create a magnetic waveguide on a chip. A current flowing in a microfabricated wire and a perpendicular bias field form an elongated microtrap. Imperfections in the wire force the current to follow a weakly meandering path and generate therefore a magnetic disorder potential along the center of the guide.

and therefore the transmission becomes a function of time. In this case we simulate the propagation process over a long period $\tau$ (ideally $\tau \rightarrow \infty$ ) and characterize the transport properties of the guide by means of the time-averaged transmission

$$
\bar{T}=\lim _{\tau \rightarrow \infty} \frac{1}{\tau} \int_{t}^{t+\tau} T\left(t^{\prime}\right) d t^{\prime} \quad(t>0) .
$$

This choice of working with the mean value $\bar{T}$ is inspired by common experimental setups: the number of condensed atoms $\mathcal{N}_{A}$ reaching the downstream region during the time $\tau$ is $\mathcal{N}_{A}=\tau \bar{T} J_{i}$. This number of atoms can be determined experimentally, e.g., by use of absorption spectroscopy.

\section{A SIMPLE MODEL OF DISORDER}

In order to compute transport properties through disordered regions in magnetic waveguides, it is necessary to introduce an appropriate model for the static random magnetic potential along the center of the waveguide. We first briefly recall the basic principle to generate elongated magnetic waveguides for cold atoms or condensates. A typical setup that is commonly implemented on atomic chips is the socalled side wire guide [33]. As sketched in Fig. 2 a circular magnetic field $\mathbf{B}_{0}$, created by an electric current $I$ that flows along a straight microfabricated quasi-two-dimensional wire, and a homogeneous bias field $\mathbf{B}_{\perp}$ form a minimum of the magnetic field parallel to the wire at distance $h$. An offset field $\mathbf{B}_{\|}$applied parallel to the wire reduces losses induced by spin-flip processes near the magnetic field minimum.

For a spatially homogeneous current density in an idealized wire, the magnetic waveguide is perfectly uniform along its longitudinal axis. In reality, however, inhomogeneities in the current density inside the wire have to be taken into account. Such deviations from a homogeneous current flow can be induced by shape fluctuations of the wire or impurities inside the metal. These imperfections cause a magnetic field roughness along the center of the waveguide that acts as an additional potential and prevents perfect transmission of condensate beam through the guide [34]. This additional magnetic-field component increases as the distance to the chip surface diminishes and is expected to reduce the transmission noticeably.

In the following we consider a steady state current density $\mathbf{j}(\mathbf{r})$ flowing in a thin quasi-two-dimensional metallic wire. Due to the wire imperfections the current density varies with the position $\mathbf{r}$. We decompose $\mathbf{j}(\mathbf{r})$ into a large constant component $\mathbf{j}_{0}$ flowing parallel to the wire and a small component $\delta \mathbf{j}(\mathbf{r})$

$$
\mathbf{j}(\mathbf{r})=j_{0} \mathbf{e}_{x}+\delta \mathbf{j}(\mathbf{r}) .
$$

At the center of the waveguide the circular magnetic field $\mathbf{B}_{0}$ that is generated by $\mathbf{j}_{0}$ cancels with the bias field $\mathbf{B}_{\perp}$. Hence, the total magnetic field along the center of the waveguide is given by

$$
\mathbf{B}(x, 0, h)=B_{\|} \mathbf{e}_{x}+\delta \mathbf{B}(x, 0, h),
$$

where $\delta \mathbf{B}=\delta B_{x} \mathbf{e}_{x}+\delta B_{y} \mathbf{e}_{y}+\delta B_{z} \mathbf{e}_{z}$ is computed from the BiotSavart law

$$
\delta \mathbf{B}=\frac{\mu_{0}}{4 \pi} \int d^{3} \mathbf{r}^{\prime} \frac{\delta \mathbf{j}\left(\mathbf{r}^{\prime}\right) \times\left(\mathbf{r}-\mathbf{r}^{\prime}\right)}{\left|\mathbf{r}-\mathbf{r}^{\prime}\right|^{3}} .
$$

The effective potential for the atoms is proportional to the modulus of the magnetic field

$$
|\mathbf{B}|=\sqrt{\left(B_{\|}+\delta B_{x}\right)^{2}+\delta B_{y}^{2}+\delta B_{z}^{2}} .
$$

As $\delta \mathbf{j}$ is supposed to be small, we keep only terms of first order in $\delta \mathbf{j}$. This yields the simple result

$$
|\mathbf{B}|=B_{\|}+\delta B_{x} .
$$

Hence, within the approximation of small current fluctuations, the disorder potential along the center of the waveguide is given by

$$
V(x)=\mu_{B} \delta B_{x}(x, 0, h) .
$$

We now consider a quasi-two-dimensional wire of length $L$ in the $x$ direction and width $w$ in the $y$ direction. A proper description of the current density $\mathbf{j}(\mathbf{r})$ in the metallic wire would require an accurate microscopic model for structural dislocations of the wire as well as its impurities [34,35]. In the present work we adopt a more simple and phenomenological approach, which is valid if the length scale $l$ on which $\mathbf{j}(\mathbf{r})$ typically fluctuates is much smaller than the height $h$ of the waveguide. To this end, we divide the wire into $\mathcal{N}$ equal blocks of length $l$ width $w$ and thickness $\Delta_{z}$ (see Figs. 2 and $3)$. For each block of volume $V$ we compute the average current density

$$
\mathbf{j}^{\nu}=\frac{1}{V} \int_{-\Delta z / 2}^{\Delta z / 2} d z \int_{(\nu-1) l}^{\nu l} d x \int_{-w / 2}^{w / 2} d y \mathbf{j}(\mathbf{r}) .
$$

(The index $\nu=1, \ldots, \mathcal{N}$ labels the blocks and the corresponding mean current densities $\mathbf{j}^{\nu}$.) The total electric current along the wire is given by 


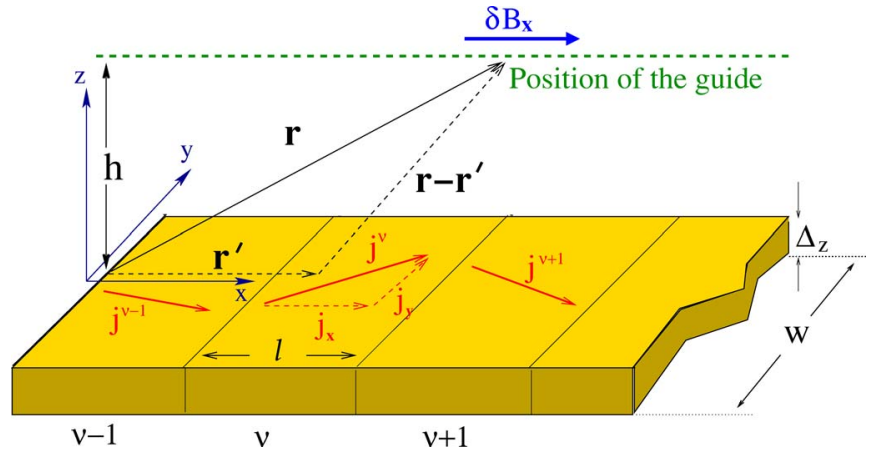

FIG. 3. (Color online) Partitioning of the wire in equal blocks of length $l$, width $w$, and thickness $\Delta_{z}$. For each block we compute an average current density $\mathbf{j}^{\nu}$. The current component parallel to the $y$ direction is at the origin of the magnetic disorder potential along the center of the waveguide.

$$
I=\int_{-\Delta_{z} / 2}^{\Delta_{z} / 2} d z \int_{-w / 2}^{w / 2} d y \mathbf{j}(\mathbf{r}) \cdot \mathbf{e}_{x}=w \Delta_{z} \mathbf{j}^{\nu} \cdot \mathbf{e}_{x}
$$

Hence, in the usual case of a stationary electric current $I$, the $x$ component of $\mathbf{j}^{\nu}$ is given by the constant value $j_{0}$ of Eq. (10) for all $\nu$, and we have

$$
\mathbf{j}^{\nu}=j_{0} \mathbf{e}_{x}+\delta j_{y}^{v} \mathbf{e}_{y}+\delta j_{z}^{\nu} \mathbf{e}_{z}
$$

The thickness $\Delta_{z}$ of the wire is assumed to be much smaller than all other relevant length scales. We therefore assume $\left|\delta j_{z}^{\nu}\right| \ll\left|\delta j_{y}^{\nu}\right|$ and neglect the contribution of $\delta j_{z}^{\nu}$ to the disorder potential in the following. This yields

$$
V(x)=\mu_{B} \sum_{\nu=1}^{\mathcal{N}} \delta B_{x}^{\nu}(x, 0, h)
$$

where the magnetic-field contribution of the $\nu$ th block at the center of the waveguide is computed from the Biot-Savart law according to

$$
\begin{aligned}
\delta B_{x}^{\nu} & =\frac{\mu_{0}}{4 \pi} \int_{(\nu-1) l}^{\nu l} d x^{\prime} \int_{-w / 2}^{w / 2} d y^{\prime} \frac{\Delta_{z} h \delta j_{y}^{\nu}}{\left[\left(x-x^{\prime}\right)^{2}+h^{2}+y^{\prime 2}\right]^{3 / 2}} \\
& =\frac{\mu_{0} \Delta_{z}}{2 \pi} \delta j_{y}^{\nu}\left[\arctan \left(\frac{w u /(2 h)}{\sqrt{u^{2}+h^{2}+w^{2} / 4}}\right)\right]_{x-\nu l}^{x-(\nu-1) l} .
\end{aligned}
$$

Within the discretization procedure just described, we can introduce disorder by assuming $\delta j_{y}^{\nu}$ as a random variable, uniformly distributed in the interval $\left[-\left(3 \sigma^{2} /\right.\right.$ $\left.2 l)^{1 / 2},\left(3 \sigma^{2} / 2 l\right)^{1 / 2}\right]$. This assumption corresponds to a zero average transverse current density $\left(\left\langle\delta j_{y}^{\nu}\right\rangle=0\right)$ with a $\delta$ correlation

$$
\left\langle\delta j_{y}^{\nu} \delta j_{y}^{\nu^{\prime}}\right\rangle=\frac{\sigma^{2}}{l} \delta_{\nu, \nu^{\prime}}
$$

and allows us to reach a well-defined regime in the limit $l$ $\rightarrow 0$. In that limit $\delta j_{y}^{\nu}$ is replaced by an $x$-dependent quantity $\delta j_{y}(x)$ verifying [36]

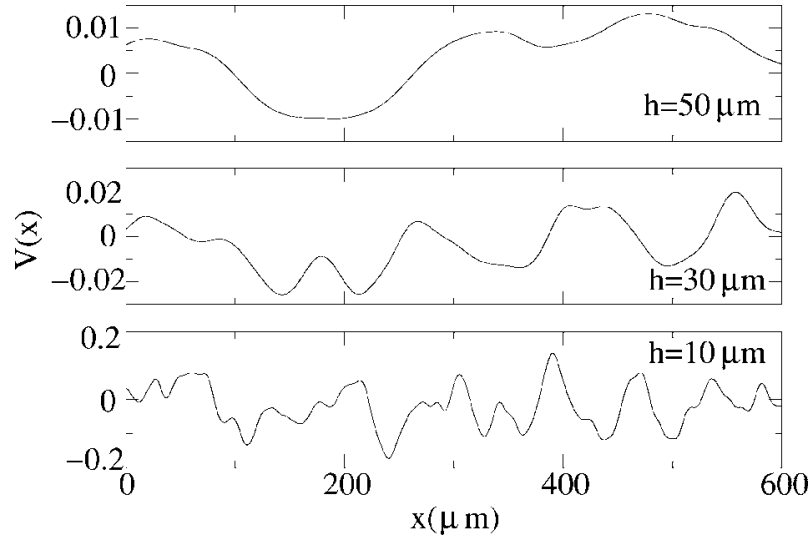

FIG. 4. Numerically computed representative examples for disorder realizations at different distances $h$ between the center of the guide and the atomic chip surface. The panels show the transition from weak to strong disorder, with decreasing distance $h$. The disorder potential is given in units of $\mu_{0} \mu_{B} w \Delta_{z} \sigma /(2 \pi)$.

$$
\left\langle\delta j_{y}(x) \delta j_{y}\left(x^{\prime}\right)\right\rangle=\sigma^{2} \delta\left(x-x^{\prime}\right) .
$$

Here, the parameter $\sigma$ fixes a scale for the typical deviation of the current density from a homogeneous current flow. Since the fluctuations in $\delta j_{y}$ are certainly proportional to $j_{0}$ $=I /\left(w \Delta_{z}\right)$, we can write $\sigma=j_{0} \sqrt{\ell^{*}}$. Here $\ell^{*}$ is a characteristic length depending on the properties of the metallic wire, which can in principle be found from experimental investigations.

Due to the convolution procedure in Eq. (20), the shortrange disorder in the electric wire induces a smoothly varying potential $V(x)$ along the guide. This is clearly visible in Fig. 4, which shows the disorder potentials that result from three numerically generated sets of current densities $j_{y}^{\nu}$, at three different heights $h$ of the waveguide. The disorder potential is smoother for large distances $h$, and becomes more rough (and its typical intensity increases) as $h$ diminishes.

One has $\langle V(x)\rangle=0$ and it is appropriate to characterize the random potential by studying the correlation function

$$
C\left(x-x^{\prime}\right)=\left\langle V(x) V\left(x^{\prime}\right)\right\rangle .
$$

In Fig. 5 we show results for $C\left(x-x^{\prime}\right)$ at different heights $h$. The correlation function is computed numerically by averaging over a large number of different disorder realizations. We find that it can be fitted with good accuracy by a Lorentzian curve

$$
C\left(x-x^{\prime}\right) \simeq \frac{\gamma l_{c}}{l_{c}^{2}+\left(x-x^{\prime}\right)^{2}} .
$$

This allows extraction of the correlation length $l_{c}$ and establishment of an empirical relation between the height $h$ and $l_{c}$. In the regime where the width $w$ of the wire and the discretization length $l$ are of the same order, we find that the correlation length depends linearly on the distance between wire and waveguide, $l_{c} \simeq s h$, with a proportionality constant $s$ that varies between 1 and 2 . For the experimentally relevant case of $w=4 \mu \mathrm{m}$ (a wire of this size has been realized by Ott et al. [11]) we find $s=1.2$. 


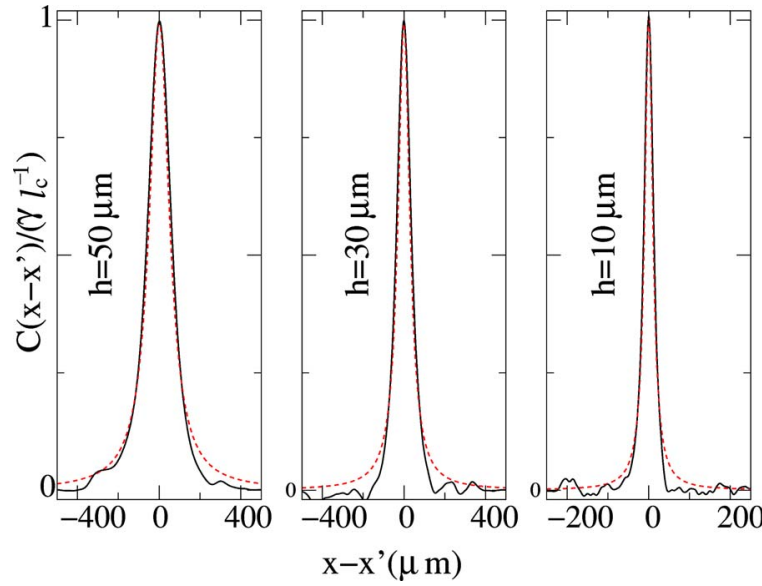

FIG. 5. (Color online) Numerically computed correlation functions for different distances $h$ between the center of the guide and the atomic chip surface (solid lines). Dashed lines: Fit to a Lorentzian curve.

Theoretically, this result may be understood as follows. In the continuous limit $l \rightarrow 0$ (and in the idealized case of an infinitely long wire), Eq. (20) takes, in the regime $w \gg h$, the particularly simple form

$$
V(x)=\frac{\mu_{B} \mu_{0}}{2 \pi} \int_{-\infty}^{+\infty} d x^{\prime} \frac{h \Delta_{z} \delta j_{y}\left(x^{\prime}\right)}{\left(x-x^{\prime}\right)^{2}+h^{2}} .
$$

In this case the disorder potential is exactly Lorentz correlated with $l_{c}=2 h$ and $\gamma=\pi^{-1}\left(\mu_{0} \mu_{B} \Delta_{z} \sigma / 2\right)^{2}$. In the opposite regime $w \ll h$, the correlation function $C\left(x-x^{\prime}\right)$ cannot be computed analytically, but its Fourier transform $C_{q}$ $=\int_{-\infty}^{+\infty} \exp (-i q x) C(x) d x$ can be calculated. One obtains

$$
C_{q}=\left(\frac{\mu_{0} \mu_{B} w \Delta_{z} \sigma}{2 \pi}\right)^{2}\left[q K_{1}(q h)\right]^{2},
$$

where $K_{1}$ is the modified Bessel function of the first kind [37]. $C_{q}$ as given in Eq. (26) is not very different from the Fourier transform of a Lorentzian (a decreasing exponential), and this is the reason why $C\left(x-x^{\prime}\right)$ can be fitted reasonably well by a Lorentzian also in the regime $h \gg w$. To find a sensible Lorentzian fit, one can for instance try to reproduce $C(0)$ and $C^{\prime \prime}(0)$ obtained from Eq. (26) with the parameters $\gamma$ and $l_{c}$ of Eq. (24): $C(0)=\gamma / l_{c}$ and $C^{\prime \prime}(0)=-2 \gamma / l_{c}^{3}$. This leads to

$$
\frac{l_{c}}{h}=\left(\frac{2 \int_{0}^{+\infty} t^{2} K_{1}^{2}(t) d t}{\int_{0}^{+\infty} t^{4} K_{1}^{2}(t) d t}\right)^{1 / 2} \simeq 1.46 .
$$

Thus, again in this limit, we find that $l_{c}$ is of the type $l_{c}$ $\simeq s h$. The important outcome of this discussion is that, for the continuous model $(l \rightarrow 0)$-in both limits $w \ll h$ and $w$ $\gg h$-and also in the numerical realizations of the disorder with a finite grid $l$, we obtain a random potential which is Lorentz-like correlated, with a correlation length $l_{c}$ that is proportional to the height $h$ of the trap above the chip, and with a proportionality constant of the order $1-2$. This is confirmed experimentally by the detailed studies presented in Ref. [34].

The model introduced in this section, where the disorder potential originates from white-noise-correlated fluctuating currents [see Eqs. (21) and (22)] corresponds physically to a dirty-wire model, in the sense that the very erratic random current density (22) can be considered as originating from the presence of impurities in the wire. We note that the white-noise current correlations lead to a disorder potential whose typical amplitude (for the continuous limit $l \rightarrow 0$, when $h \gg w)$ varies as $\left\langle V^{2}(x)\right\rangle^{1 / 2} \propto I h^{-3 / 2}$, different from the experimental finding $I h^{-2.2}$ of Kraft et al. [38]. In contrast, the model of a "clean wire with corrugated boundaries" introduced in [35] and developed in [34] yields in the case of a white-noise correlated boundary roughness a dependence of the form $I h^{-5 / 2}$, in closer agreement with the experimental findings of Ref. [38]. Note, however, that the experimental results of Estève et al. [34] point to a boundary roughness which is not white-noise correlated, and a typical disorder potential which decreases less rapidly than $h^{5 / 2}$, as found in the present study. Also the correlation function coming from the dirty-wire model is in better agreement with the experimental one determined in Ref. [34], which differs from the one resulting from a wire with a white-noise-disordered boundary (which has a correlation function verifying $C_{q=0}$ $=0[35])$. It thus appears that the simple dirty-wire model introduced in the present section allows one to construct a disordered potential $V(x)$ that captures most of the characteristics of microfabricated magnetic guides.

\section{WEAK DISORDER}

In this section we investigate the regime of weak disorder potentials and derive simple relations between the condensate density and the disorder potential $V(x)$. Weak disorder means in this context that the propagation of the condensate is only marginally affected by the scattering region. This implies that the kinetic energy per particle must be much larger than the typical intensity of the disorder potential [which can be estimated, for instance, by the standard deviation $\left\langle V^{2}(x)\right\rangle^{1 / 2}$. We shall argue below that a secondary criterion is necessary to characterize this regime, namely, that the length of the disordered region is small compared to the characteristic length scale $L_{d}$ (to be defined below) typical for the decrease of the transmission.

First, we rewrite the Gross-Pitaevskii equation (1) in the well-known form of the hydrodynamic equations

$$
\frac{\partial}{\partial t} n=-\frac{\partial}{\partial x}(n u)
$$

and

$$
m \frac{\partial u}{\partial t}=\frac{\partial}{\partial x}\left(\frac{\hbar^{2}}{2 m n^{1 / 2}} \frac{\partial^{2} n^{1 / 2}}{\partial x^{2}}-\frac{m u^{2}}{2}-V(x)-g n\right),
$$

where $u$ is the condensate velocity. In the case of a stationary state we have $\partial_{t} n=0$ and $\partial_{t} u=0$, from which it follows that the current $J=n u$ is constant. Integration of Eq. (29) then yields 


$$
\mu=V(x)+g n+\frac{J^{2}}{2 m n^{2}}-\frac{\hbar^{2}}{2 m n^{1 / 2}} \frac{\partial^{2} n^{1 / 2}}{\partial x^{2}} .
$$

This is the time-independent Gross-Pitaevskii equation for a current-carrying scattering state. In the downstream region an outgoing plane wave $\psi(x)=\sqrt{n_{0}} e^{i k x}$ is expected. The equilibrium density $n_{0}$ coincides with the supersonic solution of the dispersion relation (3). Defining $\rho(x) \equiv n(x) / n_{0}$ and $v(x) \equiv 2 m V(x) /\left(\hbar^{2} k^{2}\right)$ one may rewrite Eq. (30) in a dimensionless form:

$$
-\frac{1}{\rho^{1 / 2}} \frac{\partial^{2} \rho^{1 / 2}}{\partial x^{2}}+\frac{\rho-1}{\xi^{2}}+k^{2}\left(\frac{1}{\rho^{2}}-1+v(x)\right)=0 .
$$

In this expression we made use of the dispersion relation Eq. (3) and expressed $J=n_{0} \hbar k / m$ in terms of the downstream density $n_{0}$ and of the outgoing wave vector $k$. The quantity $\hbar^{2} k^{2} /(2 m)=\mu-g n_{0}$ is the kinetic energy of the outgoing plane wave with equilibrium density $n_{0} . \xi=\hbar / \sqrt{2 m n_{0} g}$ is the condensate healing length.

In order to find perturbative solutions of Eq. (31) for $v(x) \ll 1$, we insert the ansatz $\rho(x)=1+\delta \rho(x)$ into Eq. (31) and keep only terms that are linear in $\delta \rho(x)$ :

$$
\frac{\partial^{2}}{\partial x^{2}} \delta \rho(x)+4 \kappa^{2} \delta \rho(x)=2 k^{2} v(x),
$$

where

$$
\kappa=k \sqrt{1-\frac{1}{2 \xi^{2} k^{2}}} .
$$

The solution of Eq. (32) in the presence of the downstream boundary conditions $\delta \rho(L)=0, \delta \rho^{\prime}(L)=0$ (flat downstream density) is [40]

$$
\begin{gathered}
\delta \rho(x)=\frac{k^{2}}{\kappa} \int_{x}^{L} \sin \left[2 \kappa\left(x^{\prime}-x\right)\right] v\left(x^{\prime}\right) d x^{\prime}, \\
\delta \rho^{\prime}(x)=-2 k^{2} \int_{x}^{L} \cos \left[2 \kappa\left(x^{\prime}-x\right)\right] v\left(x^{\prime}\right) d x^{\prime} .
\end{gathered}
$$

This implies that the density profile in the upstream region $(x<0)$ deduced from the linearized Eq. (32) is of the form $n(x)=n_{0}[1+\delta \rho(x)]$ with

$$
\delta \rho(x)=\delta \bar{\rho} \cos (2 \kappa x+\theta) .
$$

The amplitude $\delta \bar{\rho}$ and the phase factor $\theta$ in Eq. (35) are determined by the disorder potential $V(x)$ via Eq. (34). The modified wave number $\kappa$ fixes the period of the density oscillations.

As we are obviously in the regime of small back reflections we adopt the method of Ref. [32] to determine the transmission coefficient in an approximative way. To this end we make the ansatz $n(x)=\left|\psi_{\text {inc }}(x)+\psi_{\text {ref }}(x)\right|^{2}$ with

$$
\psi_{\text {inc }}(x)=a \exp \{i \kappa x\}
$$

$$
\psi_{\text {ref }}(x)=b \exp \{i(\kappa x+\theta)\} .
$$

Comparing the corresponding density profile with Eq. (35), one obtains the following expressions for the amplitudes $a$ and $b[39]$ :

$$
\begin{gathered}
\frac{a^{2}}{n_{0}}=1-\frac{1}{4} \delta \bar{\rho}^{2}+O\left(\delta \bar{\rho}^{4}\right), \\
\frac{b^{2}}{n_{0}}=\frac{1}{4} \delta \bar{\rho}^{2}+O\left(\delta \bar{\rho}^{-4}\right) .
\end{gathered}
$$

It was pointed out in Ref. [32], and numerically confirmed for single- and double-barrier potentials [31], that $\psi_{\text {ref }}$ can be approximately identified with the reflected component of the condensate in the case of almost perfect transmission. This corresponds to a reflexion coefficient $R=b^{2} / a^{2}=\frac{1}{4} \delta \bar{\rho}^{2}$ $+O\left(\delta \bar{\rho}^{4}\right)$ and to a transmission coefficient which can be expressed [using Eq. (35)] as

$$
T=1-\frac{1}{4} \delta \bar{\rho}^{2}=1-\frac{1}{4}\left([\delta \rho(0)]^{2}+\frac{1}{4 \kappa^{2}}\left[\delta \rho^{\prime}(0)\right]^{2}\right) .
$$

In this final expression $\delta \rho(0)$ and $\delta \rho^{\prime}(0)$ are related to the disordered potential by means of Eq. (34). Therefore, determining the transmission $T$ for a given potential $V(x)$ amounts to compute the integrals Eq. (34).

As shown in Appendix A the above procedure allows us to determine the disorder average $\langle T\rangle$ from knowledge of the correlation function of the disorder potential. For the relevant case of a Lorentzian correlation [of the form (24)] we obtain

$$
\langle T\rangle=1-\frac{L}{L_{d}},
$$

where

$$
L_{d}=\frac{\hbar^{4} \kappa^{2}}{\pi \gamma m^{2}} e^{2 \kappa l_{c}}
$$

is the characteristic length scale for the decay of the transmission. We recall here that the above analysis is valid only in the regime $\delta \bar{\rho} \ll 1$, i.e., the linear decrease of $\langle T\rangle$ in Eq. (39) is valid only for $L \ll L_{d}$. Thus, we have to refine our definition of weak disorder: not only should the intensity of the potential be small, but also the length of the disordered region should not exceed the value $L_{d}$.

As we see from expression (40), the effect of the atomatom interaction is entirely contained within the modified wave number $\kappa$ [Eq. (33)] which describes the period of the upstream density oscillations. For repulsive atom-atom interactions, we have $\kappa<k$, which implies that the mean transmission is reduced compared to the noninteracting case. This behavior is indeed well confirmed by numerical computations based on the approach presented in Sec. V. This interaction-induced decrease of the transmission was already observed in Ref. [32] and interpreted as a lack of kinetic energy compared to the interaction-free case.

In the limit of very small correlation lengths, i.e., $\kappa l_{c}$ $\ll 1$, the disorder potential can be approximated by a whitenoise potential with correlation function $C\left(x-x^{\prime}\right) \simeq \gamma \pi \delta(x$ 
$\left.-x^{\prime}\right)$. Considering the noninteracting case $(\kappa=k)$ we recover in this regime the well-known expression $L_{d}=L_{l o c}$ $\equiv\left(\hbar^{4} k^{2}\right) /\left(\pi m^{2} \gamma\right)$ for the localization length of $\delta$-correlated disorder potentials (see, e.g., Ref. [41]).

The opposite limit $\kappa l_{c} \gg 1$ can be considered as the semiclassical regime, where the de Broglie wavelength $\lambda$ $\equiv 2 \pi / k$ of the condensate is much smaller than the correlation length $l_{c}$ of the disorder potential. In this regime, the length scale $L_{d}$ is dominated by the exponential prefactor $\exp \left(2 \kappa l_{c}\right)$, and the deviations from perfect transmission $\langle T\rangle$ $\equiv 1$ vanish exponentially fast with increasing ratio $\kappa l_{c}$. The semiclassical condition $\kappa l_{c} \gg 1$ furthermore allows us to derive a simple analytical expression for the density $n(x)$ throughout the scattering region. We start from the zerothorder solution $n(x) \equiv n_{0}$ valid for $V \equiv 0$. Then, for given $\mu$ and $J$, the density $n_{0}$ can be obtained by iteratively solving the self-consistent equation [strictly equivalent to Eq. (3)]

$$
n_{0}=\sqrt{\frac{m}{2}} J\left[\mu-g n_{0}\right]^{-1 / 2},
$$

starting, e.g., with $n_{0}=J \sqrt{m /(2 \mu)}$. This procedure guarantees convergence toward the supersonic solution of Eq. (3).

The natural generalization of Eq. (41) to the case of a small but nonvanishing potential $V(x)$ is obtained by using Eq. (30) instead of Eq. (3). This yields

$$
n=n_{0}\left(-\frac{V(x)}{\mu-g n_{0}}+\frac{\mu-g n+\left(\hbar^{2} / 2 m \sqrt{n}\right)\left(\partial^{2} / \partial x^{2}\right) \sqrt{n}}{\mu-g n_{0}}\right)^{-1 / 2},
$$

where the current $J$ was substituted by means of the dispersion relation (3). We shall now find approximate solutions of this self-consistent equation in the case of weak disorder, i.e., $|v(x)| \ll 1$, where the typical value of $V$ is much smaller than $\mu-g n_{0}$, which is the kinetic energy per particle. We emphasize that this does not imply that the nonlinear term $g n_{0}$ should be small.

The zeroth-order solution of Eq. (42) is simply the constant equilibrium density $n_{0}$. Resubstituting this constant solution into the recursive equations yields the first-order solution for the condensate density

$$
n^{(1)}(x)=\frac{n_{0}}{\sqrt{1-v(x)}} .
$$

Corrections to this first-order expression particularly arise from the quantum pressure term $\left(\hbar^{2} / 2 m \sqrt{n}\right)\left(\partial^{2} / \partial x^{2}\right) \sqrt{n}$. It can be shown, however, that the latter is suppressed by a factor $\sim 1 /\left(k l_{c}\right)^{2}$ as compared to the kinetic energy $\hbar^{2} k^{2} /(2 m)$ when $n^{(1)}(x)$ is resubstituted in Eq. (42). In the semiclassical regime $k l_{c} \gg 1$, the quantum pressure term becomes negligible, and the expression (43) represents a very good approximation to the actual density of the condensate in the scattering region. We show in Appendix B that the result (43) can be derived in a way that is directly analogous to the semiclassical WKB approach.

This result is illustrated in Fig. 6, whose lower panel shows a random potential generated with the method presented in the previous section. In the upper panel we com-

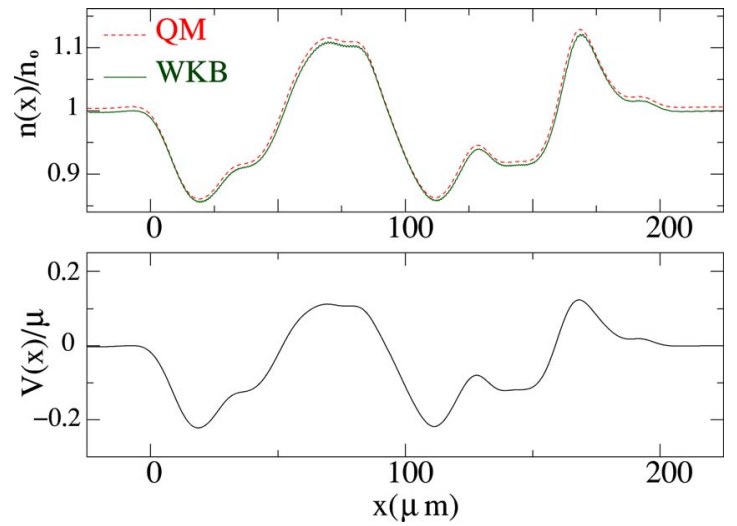

FIG. 6. (Color online) The upper panel displays a comparison of the first-order solution [WKB, Eq. (43)] with a numerically computed solution $(\mathrm{QM})$ of the Gross-Pitaevskii equation for a weakdisorder potential $V(x)$ (shown in the lower panel). The correlation length is $l_{c}=30 \mu \mathrm{m}$; the wavelength is $\lambda=3 \mu \mathrm{m}$. The ratio between interaction and kinetic energy in the incident beam is $E_{\text {int }} / E_{\text {kin }}$ $=1 / 10$.

pare the result of Eq. (43) with an exact, i.e., numerically computed solution of the Gross-Pitaevskii equation. Excellent agreement between the first-order solution and the exact solution is found. We note here that it is quite natural to find that the density profile mirrors the potential because we are dealing with current-carrying states: the relation (30) (in the absence of quantum correction) $\mu=m u^{2} / 2+V(x)+g n$ predicts that the condensate velocity becomes minimal close to the maxima of the disorder potential. It follows then from the continuity equation $J=n u=$ const that the density $n$ assumes its maxima when the velocity becomes minimal.

It is instructive to realize that classically forbidden back reflections can be taken into account by inserting the ansatz $n(x)=n^{(1)}(x)+\delta n(x)$ into Eq. (30) and linearizing the resulting equation for small $\delta n(x) / n_{0}$. To the lowest nonvanishing order in $v$, we again obtain the result (39) for the mean transmission.

Finally we consider experimental realizations of waveguides on atom chips. Typical distances $h$ between the chip surface and the guide are in the range 20-100 $\mu \mathrm{m}$. Typical disorder correlation lengths are of the same order as $h$. In recent transport experiments [43] the velocity of propagating ${ }^{87} \mathrm{Rb}$ condensates is of the order of a few millimeters per second, resulting in a mean wavelength of a few micrometers. This corresponds to the regime $\kappa l_{c} \gg 1$ withfrom Eq. (40) - a very large value of $L_{d}$. Hence the regime of weak disorder is presently the most relevant one; the kinetic energy is much larger than the typical intensity of the disordered potential and $L_{d}$ is large compared to the typical length of the disordered region; one thus expects almost perfect transmission.

\section{MODERATE AND STRONG DISORDER}

In Sec. IV we focused on weak-disorder potentials, in the limit of small reflection. The analysis was done in the regime $\mu \gg\left\langle V^{2}(x)\right\rangle^{1 / 2}$ and $L \ll L_{d}$. In the present section we still par- 
tially satisfy the first of these inequalities, but drop the second one. We will see that the behavior of the system is quite different, ranging from a regime of localization (in the limit of weak interaction) to a time-dependent behavior for larger interaction, with a power-law decay of the time-averaged transmission.

First, we shall discuss some elementary differences between the scattering problem in linear quantum mechanics and the nonlinear Gross-Pitaevskii equation. In linear quantum mechanics $(g=0)$ one finds for any scattering potential a unique stationary scattering state that is dynamically stable, and the associated transmission coefficient $T$ relates the constant incident current $J_{i}$ one to one with the transmitted current $J_{t}$. For the nonlinear Gross-Pitaevskii equation the transmission $T$ depends on the density of the propagated condensate and thereby on the current. Additionally, the phenomenon of multistability may arise. This means that for a given incident current $J_{i}$ two or more scattering states with different transmissions can coexist.

In principle all stationary scattering states that are associated with a given incident current $J_{i}$ can be found by integrating the time-independent Gross-Pitaevskii equation (8) from the downstream to the upstream region. A systematic variation of the downstream current $J_{t}$ allows one to select the desired states. This procedure, however, does not reveal any information about their dynamical stability properties, which are crucial for answering the question whether an incident condensate beam populates a stationary scattering state or not. For instance, in the case of coherent condensate transport through a double-barrier potential, three possible scattering states are expected close to the resonances, but only one of them is dynamically stable [15]. Here the advantage of integrating the time-dependent Gross-Pitaevskii equation becomes apparent: If this integration converges to a stationary scattering state we know automatically that this state is dynamically stable (otherwise small numerical deviations would exponentially increase with propagation time).

We consider an ensemble of $N$ disorder realizations with randomly varying sample lengths $L$ that are uniformly distributed between 0 and a maximal sample length. For each realization (labeled with index $\alpha$ ) we numerically compute the time evolution of the wave function and extract either the time-independent transmission $T_{\alpha}$ [if $\psi(x, t)$ converges to a stationary state] or the time-averaged transmission $\bar{T}_{\alpha}$ [if $\psi(x, t)$ remains time dependent]. For the sake of definiteness and due to its experimental relevance we consider the propagation of condensed ${ }^{87} \mathrm{Rb}$ atoms (whose scattering length is $a_{s c}=5.77 \mathrm{~nm}$ ). Our numerical computations were performed for a guide with radial trapping frequency $\omega_{\perp}=2 \pi$ $\times 100 \mathrm{~s}^{-1}$ (oscillator length $a_{\perp}=1 \mu \mathrm{m}$ ). The disorder is generated as in the previous section. The regime of strong disorder is reached by choosing a rather short distance $h$ $=5 \mu \mathrm{m}$ between the center of the guide and the chip surface, which corresponds to a correlation length $l_{c}=6 \mu \mathrm{m}$. In order to avoid excitations of the condensate into higher transversal modes we adjust the standard deviation of the potential (which is a measure of the mean potential height) to $\left\langle V^{2}(x)\right\rangle^{1 / 2} \simeq 0.12 \hbar \omega_{\perp}$. In all the following numerical calculations we consider an incident monochromatic beam with cur-
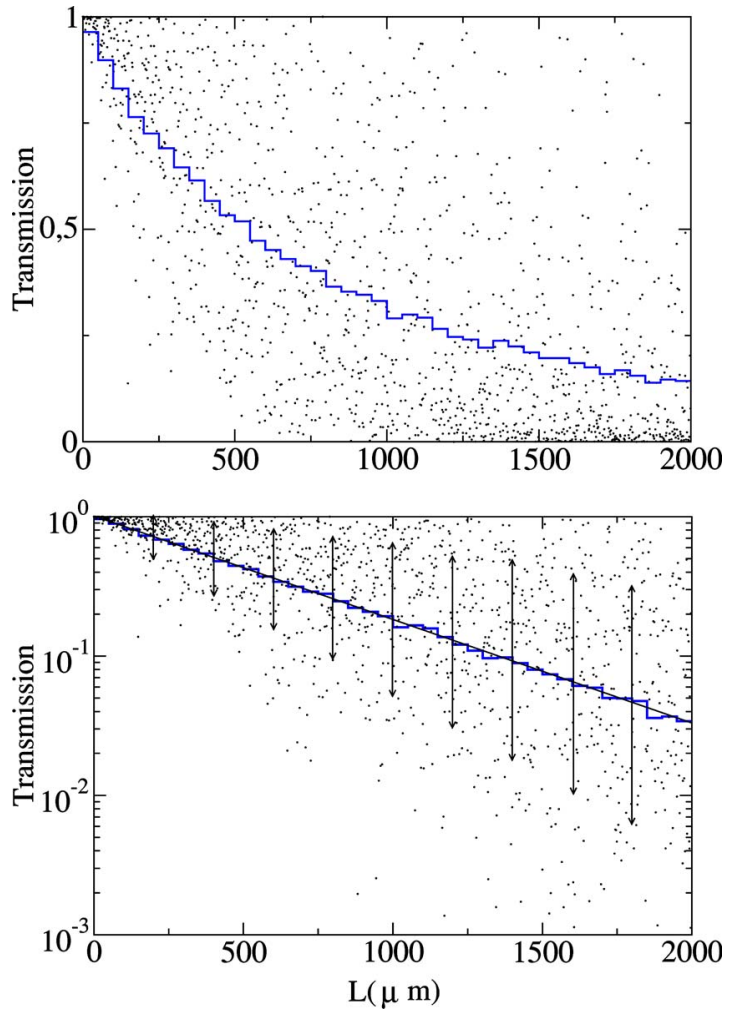

FIG. 7. (Color online) Transmission through a disordered sample as a function of sample length $L$ for the noninteracting case. Each point corresponds to a different realization of the disordered potential. Upper panel: arithmetically averaged transmission (blue staircase function). Lower panel: The geometric averaged transmission (blue staircase function) decreases exponentially with increasing $L$, as revealed by the fit with $L_{l o c}=586 \mu \mathrm{m}$ (straight black line). The arrows mark the logarithmic standard deviation.

rent $J_{i}=10^{3}$ atoms per second and wavelength $\lambda=10 \mu \mathrm{m}$. Then the chemical potential is $\mu=0.25 \hbar \omega_{\perp}$ (in the linear case the chemical potential takes the slightly different value $\left.\mu=0.23 \hbar \omega_{\perp}\right)$.

It is instructive to focus first on the linear case $(g=0)$ which has already been extensively investigated in the context of localization theory [22,41]. In the localized regime the transmission decays exponentially with increasing system length $L$, i.e., $\langle T\rangle=\exp \left(-L / L_{l o c}\right)$ where $L_{l o c}$ is the socalled localization length. The points in the upper panel of Fig. 7 mark for each disorder realization the associated transmission $T_{\alpha}(L)$ as a function of the sample length $L$. To extract from these data a characteristic scaling law for the $L$ dependence of the transmission we divide $L$ into equal intervals of length $\Delta L \ll L$. We then compute the mean transmission at sample length $L$ by summing up all the values $T_{\alpha}$ corresponding to a sample length lying in the interval of width $\Delta L$ centered at $L$ :

$$
\langle T\rangle_{a}(L)=\frac{1}{N_{L}} \sum_{\alpha} T_{\alpha}\left(L^{\prime}\right), \quad L-\frac{\Delta L}{2}<L^{\prime}<L+\frac{\Delta L}{2} .
$$

$N_{L}$ is the number of samples in the interval under consideration. 


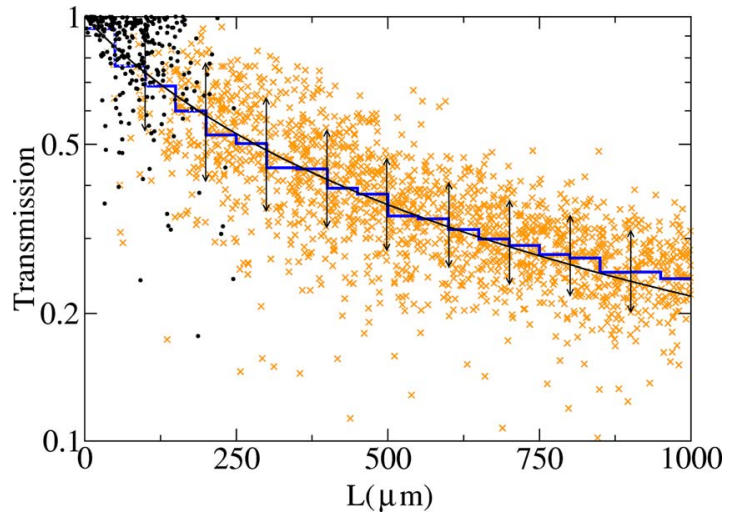

FIG. 8. (Color online) Transmissions through an ensemble of disorder realizations for a moderate nonlinearity $E_{\text {int }} / E_{\text {kin }}=1 / 10$; the characteristics of the incident beam are given in the main text. A transition from a time-independent to a time-dependent regime is observed at critical length $L^{*} \simeq 125 \mu \mathrm{m}$. The black dots represent the transmissions in the time-independent regime and the (orange online) crosses are the time-averaged transmissions in the timedependent regime. The staircase function is the geometrically averaged transmission. It is well approximated by the algebraic scaling law $L_{0} /\left(L+L_{0}\right)$ (smooth solid line) with $L_{0}=287 \mu \mathrm{m}$.

The step function in Fig. 7 shows the decrease of $\langle T\rangle_{a}$ for 30000 disorder realizations and $\Delta L=50$ (for the sake of clarity we show only 2000 points in the plot). In the context of localization theory it is convenient to investigate scaling laws by means of the geometrically averaged transmission

$$
\langle T\rangle_{g}=e^{\langle\ln (T)\rangle}, \quad\langle\ln (T)\rangle=\frac{1}{N_{L}} \sum_{\alpha} \ln \left[T_{\alpha}\left(L^{\prime}\right)\right],
$$

because, contrary to $\langle T\rangle_{a}$, the average $\langle\ln (T)\rangle$ is a selfaveraging quantity of the system $[41,42]$. The lower panel of Fig. 7 shows $\langle T\rangle_{g}$, which follows clearly an exponential law. This is clear evidence for the appearance of localization. We can extract the localization length, which here is $L_{l o c}$ $=586 \mu \mathrm{m}$. We note the wide spread of the data points around their average. This spread is quantified by the logarithmic standard deviation

$$
\Delta \ln (T)=\left(\sqrt{\frac{1}{N_{L}} \sum_{\alpha}\left[\ln \left(T_{\alpha}\right)-\langle\ln (T)\rangle\right]^{2}}\right),
$$

which is shown as arrows in the lower panel of in Fig. 7. We find an almost linear increase of $\Delta \ln (T)$ with the sample length.

Is the conventional localization scenario, with the characteristic exponential decrease of the transmission $[16,17]$, still valid in the case of interacting atoms? To address this question we now calculate the transport in presence of a moderate nonlinearity where the ratio of interaction and kinetic energy in the incident beam is $E_{\text {int }} / E_{\text {kin }} \simeq 1 / 10$. Contrarily to the linear case, time-dependent behavior becomes now a dominant feature as shown in Fig. 8. We find that dynamical stable scattering states (black dots in Fig. 8) are populated only for sample lengths that are smaller than a critical length $L^{*}$, which is here of order of $125 \mu \mathrm{m}$. For samples with length $L \geqq L^{*}$ we find a crossover region where timedependent behavior sets in and convergency to a stationary state is achieved for only a certain fraction of disorder samples. $\psi(x, t)$ remains time dependent (orange crosses) for all samples when we reach the regime where $L$ is notably larger than $L^{*}$. In the time-dependent case the data points display the time-averaged transmissions $\bar{T}_{\alpha}(9)$.

In order to extract a scaling law from our data, we compute the ensemble-averaged transmission [in the timedependent cases $T_{\alpha}$ in Eqs. (44)-(46) is replaced by $\left.\bar{T}_{\alpha}\right]$. We find that the geometrically averaged transmission $\langle T\rangle_{g}$ (step function in Fig. 8) decreases inversely with the sample length $L$ and is well approximated by the algebraic function (smooth line in Fig. 8)

$$
\langle T\rangle_{g}=\frac{L_{0}}{L+L_{0}}
$$

with the decay length $L_{0}$. Such a scaling law is characteristic for transport in systems with loss of phase coherence between the single-scattering events. Indeed, if one considers a series of successive scatterers and calculates the transmission by neglecting all interference effects, one derives exactly the scaling law of Eq. (47) $[44,45]$. Such an Ohmic behavior is observed for electron transport through mesoscopic metal structures in the limit of small dephasing lengths $[44,46]$.

Another striking feature is the distribution of the data points in Fig. 8. Contrarily to the linear case, this distribution is now clearly restricted to the neighborhood of the average transmission, and the standard deviation $\Delta T(L)$ decreases for long sample lengths $L$. Hence, in the regime of large lengths one expects to find the $\bar{T}_{\alpha}$ 's in a narrow interval centered around the averaged transmission. Loosely speaking, $\bar{T}_{\alpha}$ becomes more or less sample independent. For the sake of completeness we mention that ideally the time averages $\bar{T}_{\alpha}$ should be computed for an infinitely long period. Of course this cannot be done numerically, but we verified that the averaged transmission and the standard deviation do not change if we increase in Eq. (9) the averaging time window from $\tau$ to $2 \tau$ and $3 \tau$.

The above presented computations demonstrate that even a moderate nonlinearity leads to a dramatic change of the transmission properties. In particular, the usual interpretation of the transmission behavior in terms of localization is put in question in the case of interacting particles. In order to obtain deeper insight into that matter, we redo the above computation with a very weak nonlinearity, such that $E_{\text {int }} / E_{\text {kin }}$ $=1 / 100$. Figure 9 shows that for this case the crossover from time-independent to time-dependent behavior is shifted to larger sample lengths $(L \gtrsim 600 \mu \mathrm{m})$. This indicates the existence of a critical nonlinearity beyond which the system exhibits time dependence. Indeed, preliminary studies show that for each disorder sample length $L$ there is a critical value $g^{*}$ above which no stationary scattering state can be populated, or, equivalently, for each strength of interaction $g$, there is a critical disorder length $L^{*}$ above which the flow is time dependent. We find that $L^{*}$ decreases with increasing nonlinearity. This is the reason why stationary states can be 


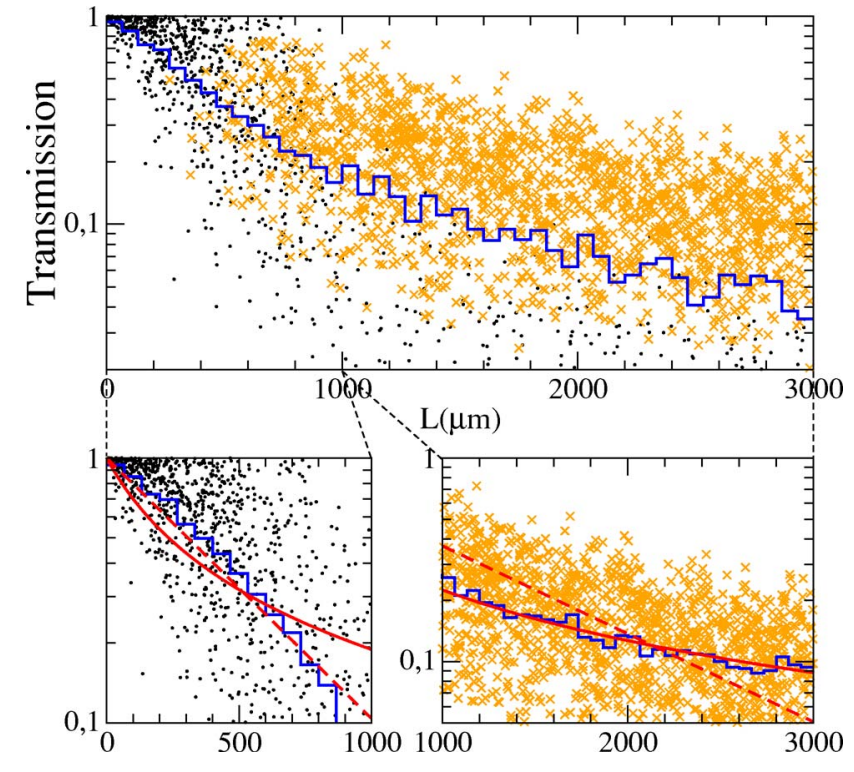

FIG. 9. (Color online) Transition from time-independent to time-dependent behavior in presence of a very weak nonlinearity $\left(E_{i n t} / E_{k i n}=1 / 100\right)$. The staircase function in the upper panel shows the geometric average of both time-independent and timedependent transmissions (black dots and orange crosses, respectively). The lower panels display separate averages over the timeindependent transmissions (lower left panel) and the timedependent transmissions (lower right panel) and show the best exponential (dashed line, colored red online) as well as algebraic (solid line, colored red online) fits to the data. Clearly, the timeindependent transmissions decrease exponentially with increasing $L$ (with localization length $L_{l o c}=439 \mu \mathrm{m}$ ) while the averaged transmissions in the time-dependent case decay according to Ohm's law.

populated in short but not in long disorder regions and why the crossover to time-dependent dynamics in Fig. 9 is displaced to larger sample lengths $L$ compared with Fig. 8 .

From Fig. 9 we also see that the Ohmic decrease of the transmission is intimately connected to the occurrence of time-dependent dynamics. This is clearly indicated by the fact that the time-independent data points can be accurately fitted by an exponential law, and not by an algebraic one [47]. We infer from this observation that as long as stationary states are populated the system follows the conventional scenario of localization even in the presence of repulsive atomatom interactions, with a smaller localization length than in the interaction-free case. This scenario seems to break down as soon as the scattering process of the condensate becomes intrinsically time dependent. We tentatively attribute this phenomenon to the fact that the definition of the mean transmission involves a time average over the propagation process [see Eq. (9)]. Therefore, information about the phase coherence, which is in principle preserved by the timedependent Gross-Pitaevskii equation, becomes lost in the time-averaging procedure.

\section{CONCLUSIONS}

In this paper we have presented a study of transport of Bose-Einstein condensates in the presence of disorder. We introduced a one-dimensional model for the disorder potential in the case of a condensate that propagates through a magnetic waveguide over a microchip. We assumed for this model that the transverse current density in the microfabricated wire exhibits a white-noise correlation. We showed that this yields a disorder potential that is Lorentz-like correlated along the axis of the waveguide.

In the regime of weak disorder, a perturbative approach allowed us to estimate the deviations from perfect transmission. We found that on length scales much smaller than a characteristic length $L_{d}$-which is determined by the correlation length of the disorder potential and the healing length of the condensate - the transmission decreases linearly with increasing length $L$ of the disorder region. The presence of a repulsive atom-atom interaction diminishes the transmission compared to the interaction-free case. Furthermore, in the limit of large correlation lengths $\left(l_{c} \gg \lambda\right)$ we could identify a semiclassical regime where the backscattering is exponentially suppressed and where the condensate density mirrors the shape of the disorder potential $V(x)$.

The numerical approach presented in Sec. II provides access also to the regime of moderate- and strong-disorder potentials and allows simulation of a realistic transport process. In the case of noninteracting atoms we find clear evidence of the appearance of localization. In the presence of interaction, the time dependence of the transmissions becomes a dominant feature of the system. We find that stationary scattering states can then be populated only in waveguides with rather short disorder regions, whereas the condensate exhibits a strongly time-dependent dynamics if we consider large sample lengths. Our numerical calculations show that the critical length $L^{*}$, at which the crossover between the two different regimes occurs, is shifted toward shorter sample lengths when the strength of the interaction is increased. It remains an open problem to determine this critical length $L^{*}$ analytically from the system-specific parameters, which are the incident current, the kinetic energy, the average height of the disorder potential, and the associated correlation length.

Our numerical study was restricted to Lorentz-correlated disorder in the atom-chip context. We expect, however, no significant differences for other types of disorder potentials, such as speckle fields or point scatterers. Indeed, preliminary studies on the transport of BECs in presence of randomly placed $\delta$-like barriers reveal qualitatively the same phenomenology: A regime of time-dependent scattering sets in beyond a critical interaction strength (or sample length), and the transmission decreases according to an Ohm-like law rather than to an exponential one. An important aspect that remains unexplored, on the other hand, is the depletion of the condensate, due to atom-atom scattering events, and the appearance of a thermal cloud that propagates together with the condensate. This issue should be rather relevant in the regime of time-dependent scattering, but cannot be studied with our present approach, which is based on the mean-field description of the condensate. Since the interaction with such a thermal cloud will lead to an additional cause for incoherent transport, we expect no qualitative change as far as the Ohmic power-law decay of the transmission is concerned.

The present work opens further perspectives for the study of transport in phase-coherent systems. In particular the re- 
pulsion between the atoms leads to a behavior different from the one expected in the noninteracting and attractive cases. Throughout this work we have considered realistic values of the parameters (intensity and correlation length of the potential, distance from the guide to the microchip, incident current of the beam) describing a BEC of ${ }^{87} \mathrm{Rb}$ in a waveguide, and hope to motivate experimental studies testing the results presented in this work.

\section{ACKNOWLEDGMENTS}

It is a pleasure to thank Isabelle Bouchoule, Ignacio Cirac, Carsten Henkel, Markus Popp, and Dirk Witthaut for fruitful and inspiring discussions. We acknowledge the financial support of the Bayerisch-Französchisches Hochschulzentrum (BFHZ), from the Deutsche Forschungsgemeinschaft (within the Research School GRK 638), and from CNRS and Ministère de la Recherche (Grant ACI Nanoscience 201). Laboratoire de Physique Théorique et Modèles Statistiques is Unité Mixte de Recherche de l'Université Paris XI et du CNRS, UMR 8626.

\section{APPENDIX A}

In this appendix we derive a relation between the mean transmission $\langle T\rangle$ and the correlation function of the disorder potential in the weak-disorder limit. Taking the mean value of Eq. (38) gives

$$
\langle T\rangle=1-\frac{1}{4}\left(\left\langle[\delta \rho(0)]^{2}\right\rangle+\frac{1}{4 \kappa^{2}}\left\langle\left[\delta \rho^{\prime}(0)\right]^{2}\right\rangle\right) .
$$

Therefore the problem of computing $\langle T\rangle$ reduces to the calculation of the averaged values

$$
\begin{aligned}
\left\langle[\delta \rho(0)]^{2}\right\rangle & =\frac{1}{N} \sum_{i=1}^{N}\left[\delta \rho_{i}(0)\right]^{2}, \\
\left\langle\left[\delta \rho^{\prime}(0)\right]^{2}\right\rangle & =\frac{1}{N} \sum_{i=1}^{N}\left[\delta \rho_{i}^{\prime}(0)\right]^{2} .
\end{aligned}
$$

Equation (34) allows us to write these averages as

$$
\begin{gathered}
\left\langle[\delta \rho(0)]^{2}\right\rangle=\frac{k^{4}}{\kappa^{2}} \int_{0}^{L} d x \sin (2 \kappa x) \int_{0}^{L} d x^{\prime} \sin \left(2 \kappa x^{\prime}\right)\left\langle v(x) v\left(x^{\prime}\right)\right\rangle, \\
\left\langle\left[\delta \rho^{\prime}(0)\right]^{2}\right\rangle= \\
4 k^{2} \int_{0}^{L} d x \cos (2 \kappa x) \int_{0}^{L} d x^{\prime} \cos \left(2 \kappa x^{\prime}\right) \\
\times\left\langle v(x) v\left(x^{\prime}\right)\right\rangle,
\end{gathered}
$$

where $\left\langle v(x) v\left(x^{\prime}\right)\right\rangle$ is the correlation function of the potential. We evaluate $\langle T\rangle$ for the particularly interesting cases of a $\delta$-correlated white-noise potential and a Lorentziancorrelated disorder potential. In the first case the correlation function reads

$$
\left\langle v(x) v\left(x^{\prime}\right)\right\rangle=\gamma\left(\frac{2 m}{\hbar^{2} k^{2}}\right)^{2} \pi \delta\left(x-x^{\prime}\right),
$$

and the integrals in Eq. (A3) can be easily evaluated by means of integration by parts. This yields

$$
\begin{gathered}
\left\langle[\delta \rho(0)]^{2}\right\rangle=\frac{2 m^{2} \gamma \pi}{\hbar^{4} \kappa^{2}} L+c, \\
\left\langle\left[\delta \rho^{\prime}(0)\right]^{2}\right\rangle=4 \kappa^{2}\left\langle[\delta \rho(0)]^{2}\right\rangle,
\end{gathered}
$$

where $c$ is a dimensionless constant which is of the order $\left(4 \gamma \pi m^{2}\right) /\left(\hbar^{4} \kappa^{3}\right)$. For sample lengths $L \gg \kappa^{-1}$ we can neglect $c$ and we keep only terms that scale linearly with $L$. By using this approximation we find with Eq. (A1) for a white-noise random potential

$$
\langle T\rangle=1-\frac{\pi m^{2} \gamma L}{\hbar^{4} \kappa^{2}} .
$$

In the case of a Lorentzian correlation,

$$
\left\langle v(x) v\left(x^{\prime}\right)\right\rangle=\gamma\left(\frac{2 m}{\hbar^{2} k^{2}}\right)^{2} \frac{l_{c}}{l_{c}^{2}+\left(x-x^{\prime}\right)^{2}},
$$

an exact analytic evaluation of the integrals (A3) is not possible. Nevertheless, in the regime $L \gg l_{c}$ and $L \gg 1 / \kappa$ the inner integral can be approximated with high accuracy by the real or, respectively, the imaginary part of

$$
\int_{0}^{L} \frac{1}{\pi} \frac{l_{c} e^{2 i \kappa x^{\prime}} d x^{\prime}}{\pi l_{c}^{2}+\left(x-x^{\prime}\right)^{2}} \simeq e^{2 \kappa\left(i x-l_{c}\right)}[\Theta(x)-\Theta(x-L)] .
$$

The same integration by parts procedure as in the whitenoise potential yields a result that is only modified by the occurrence of the exponential factor $\exp \left(-2 \kappa l_{c}\right)$. Therefore, in presence of a Lorentzian correlation the mean transmission reads

$$
\langle T\rangle=1-\frac{\pi m^{2} \gamma L}{\hbar^{4} \kappa^{2}} e^{-2 \kappa l_{c}}
$$

\section{APPENDIX B}

In this appendix we demonstrate that the first-order solution Eq. (43) can also be derived from a standard WKB method [48]. Inserting the WKB ansatz $\psi(x)=\exp [i f(x)]$ [where $f(x)$ is a complex-valued function] in the timeindependent Gross-Pitaevskii equation (8) gives

$$
-\frac{\hbar^{2}}{2 m}\left[i f^{\prime \prime}-\left(f^{\prime}\right)^{2}\right]+V(x)+g e^{i\left(f-f^{*}\right)}=\mu .
$$

The potential $V(x)$ is supposed to be small compared to $\mu$ $-g n_{0}$, and its correlation length is large compared to the de Broglie wavelength. Equation (B1) can be rewritten in terms of a self-consistent equation

$$
f^{\prime}=\sqrt{\frac{2 m}{\hbar^{2}}\left[\mu-V(x)-g e^{i\left(f-f^{*}\right)}\right]+i f^{\prime \prime}},
$$

which allows us to compute recursively the unknown function $f(x)$. Treating $V(x)$ as a small perturbation motivates the use of a plane wave with wave number $k$ and density $n_{0}$ as zeroth-order approximation,

$$
f^{(0)}=k x-i \ln \left(\sqrt{n_{0}}\right) .
$$

Hence, by use of Eq. (B2), we find 


$$
f^{(1) \prime}=\frac{1}{\hbar} \sqrt{2 m\left[\mu-V(x)-g n_{0}\right]} \equiv \frac{1}{\hbar} \widetilde{P}(x),
$$

and the first-order approximation of the wave function reads

$$
\psi^{(1)}(x)=\sqrt{n_{0}} \exp \left(i \int_{a}^{x} \frac{1}{\hbar} \tilde{P}\left(x^{\prime}\right) d x^{\prime}\right) .
$$

The lower bound $a$ of the integral lies in the downstream region, where we assume the flat condensate density $n_{0}$. Inserting the second derivative

$$
f^{(1) \prime \prime}=-\frac{m}{\hbar \widetilde{P}(x)} V^{\prime}(x)=\frac{1}{\hbar} \widetilde{P}^{\prime}(x)
$$

into the self-consistent Eq. (B2) yields the second-order approximation

$$
f^{(2) \prime}=\frac{\widetilde{P}(x)}{\hbar^{2}} \sqrt{1+i \hbar \widetilde{P}^{\prime}(x) /[\widetilde{P}(x)]^{2}} \simeq \frac{\widetilde{P}(x)}{\hbar}+i \frac{\widetilde{P}^{\prime}(x)}{2 \widetilde{P}(x)} .
$$

By integrating we find the second-order approximation of the wave function

$$
\psi^{(2)}(x)=\sqrt{n_{0}} \sqrt{\frac{\tilde{P}(a)}{\tilde{P}(x)}} \exp \left(i \int_{a}^{x} \frac{1}{\hbar} \widetilde{P}\left(x^{\prime}\right) d x^{\prime}\right) .
$$

Hence, the density found with this WKB method is

$$
n(x)=\frac{\widetilde{P}(a)}{\tilde{P}(x)}=\frac{n_{0}}{\sqrt{1-v(x)}}
$$

where $v(x) \equiv V(x) /\left(\mu-g n_{0}\right)=V(x) 2 m /\left(\hbar^{2} k^{2}\right)$. Indeed, Eq. (B9) coincides exactly with the result (43) found in Sec. IV.
[1] L. Deng et al., Nature (London) 398, 218 (1999).

[2] L. Khaykovich et al., Science 296, 1290 (2002); K. E. Strecker, G. B. Partridge, A. G. Truscott, and R. G. Hulet, Nature (London) 417, 150 (2002).

[3] S. Burger et al., Phys. Rev. Lett. 83, 5198 (1999); J. Denschlag et al., Science 287, 97 (2000); Z. Dutton, M. Budde, C. Slowe, and L. V. Hau, ibid. 293, 663 (2001).

[4] L. Fallani et al., Phys. Rev. Lett. 93, 140406 (2004).

[5] D. Witthaut, M. Werder, S. Mossmann, and H. J. Korsch, Phys. Rev. E 71, 036625 (2005).

[6] M. Greiner et al., Nature (London) 415, 39 (2002).

[7] J. R. Abo-Shaeer, C. Raman, J. M. Vogels, and W. Ketterle, Science 292, 4749 (2000).

[8] M. R. Andrews et al., Phys. Rev. Lett. 79, 553 (1997).

[9] T. Bourdel et al., Phys. Rev. Lett. 93, 050401 (2004).

[10] R. Folman et al., Phys. Rev. Lett. 84, 4749 (2000).

[11] H. Ott, J. Fortagh, G. Schlotterbeck, A. Grossmann, and C. Zimmermann, Phys. Rev. Lett. 87, 230401 (2001).

[12] W. Hänsel et al., Nature (London) 413, 498 (2001).

[13] J. H. Thywissen, R. M. Westervelt, and M. Prentiss, Phys. Rev. Lett. 83, 3762 (1999).

[14] I. Carusotto and G. C. La Rocca, Phys. Rev. Lett. 84, 399 (2000); I. Carusotto, Phys. Rev. A 63, 023610 (2001).

[15] T. Paul, K. Richter, and P. Schlagheck, Phys. Rev. Lett. 94, 020404 (2005).

[16] P. W. Anderson, Phys. Rev. 109, 1492 (1958).

[17] Anderson Localization, edited by T. Ando and H. Fukuyama, Springer Proceedings in Physics Vol. 28 (Springer, Heidelberg, 1988).

[18] J. Fortágh, H. Ott, S. Kraft, A. Günther, and C. Zimmermann, Phys. Rev. A 66, 041604(R) (2002); A. E. Leanhardt et al., Phys. Rev. Lett. 89, 040401 (2002).

[19] J. E. Lye et al., Phys. Rev. Lett. 95, 070401 (2005); C. Fort et al., e-print cond-mat/0507144.

[20] D. Clément et al., Phys. Rev. Lett. 95, 170409 (2005).

[21] C. Menotti and S. Stringari, Phys. Rev. A 66, 043610 (2002).

[22] S. A. Gredeskul and Y. S. Kivshar, Phys. Rep. 216, 1 (1992).
[23] P. Devillard and B. Souillard, J. Stat. Phys. 43, 423 (1986).

[24] R. Knapp, G. Papanicolaou, and B. White, J. Stat. Phys. 63, 567 (1991).

[25] D. S. Petrov, D. M. Gangardt, and G. V. Shlyapnikov, J. Phys. IV 116, 5 (2004).

[26] M. Olshanii, Phys. Rev. Lett. 81, 938 (1998).

[27] A. D. Jackson, G. M. Kavoulakis, and C. J. Pethick, Phys. Rev. A 58, 2417 (1998).

[28] P. Leboeuf and N. Pavloff, Phys. Rev. A 64, 033602 (2001).

[29] This has been shown in Ref. [28] to be the appropriate boundary condition for our problem.

[30] T. Shibata, Phys. Rev. B 43, 6760 (1991).

[31] T. Paul, K. Richter, and P. Schlagheck (unpublished).

[32] P. Leboeuf, N. Pavloff, and S. Sinha, Phys. Rev. A 68, 063608 (2003).

[33] R. Folman et al., Adv. At., Mol., Opt. Phys. 48, 263 (2002).

[34] J. Estève et al., Phys. Rev. A 70, 043629 (2004); T. Schumm et al., Eur. Phys. J. D 32, 171 (2005).

[35] D.-W. Wang, M. D. Lukin, and E. Demler, Phys. Rev. Lett. 92, 076802 (2004)

[36] All this procedure remains perfectly valid if, instead of a uniform distribution, $\delta j_{y}^{\nu}$ is chosen to be Gaussian distributed around zero, with a standard deviation $\sigma / \sqrt{\ell}$.

[37] Handbook of Mathematical Functions, edited by M. Abramowitz and I. Stegun (Dover Publications, New York, 1972).

[38] S. Kraft, A. Günther, H. Ott, D. Wharam, C. Zimmermann, and J. Fortágh, J. Phys. B 35, L469 (2002).

[39] It is, however, important to realize that this analysis is valid only in the limit $k \xi \gg 1$. This is due to the fact that only in this limit does the total current computed from Eqs. (36) and (37) assume the correct value $J_{t}=n_{0} \hbar \mathrm{k} / \mathrm{m}$.

[40] Equation (32) is the well-known equation of a driven harmonic oscillator. $\delta \rho(x)$ is computed by use of the harmonic oscillator's Green's function.

[41] I. M. Lifshits, S. A. Gredeskul, and L. A. Pastur, Introduction to the Theory of Disordered Systems (Wiley-Interscience, New 
York, 1988).

[42] P. W. Anderson, D. J. Thouless, E. Abrahams, and D. S. Fisher, Phys. Rev. B 22, 3519 (1980).

[43] J. Forthágh, H. Ott, G. Schlotterbeck, and C. Zimmermann, Appl. Phys. Lett. 81, 1146 (2002).

[44] S. Datta, Electronic Transport in Mesoscopic Systems (Cambridge University Press, Cambridge, U.K., 1995).

[45] M. V. Berry and S. Klein, Eur. J. Phys. 18, 222 (1997).
[46] D. K. Ferry and S. M. Goodnick, Transport in Nanostructures (Cambridge University Press, Cambridge, U.K., 1997).

[47] This fact is not obvious when looking at Fig. 2, because in this figure the extent of the time-dependent region is too small to have an influence on the fit.

[48] L. D. Landau and E. M. Lifshitz, Quantum Mechanics: NonRelativistic Theory (Pergamon, Oxford, 1958). 